\title{
Deep Neck Flexor and Sternocleidomastoid Muscle Thickness Change in Persons with No Current Neck Pain using Rehabilitative Ultrasonograpic Imaging
}

\author{
Hae-Jung Lee, Ju-Min Song \\ Department of Physical Therapy, Silla University, Busan, Korea
}

\begin{abstract}
Purpose: The purpose of the study was to investigate the thickness of deep neck flexors (DNF) and sternocleidomastoid muscle (SCM) bilaterally during deep neck flexor endurance test using ultrasonography images.

Methods: Healthy volunteers $(n=22), 20-25$ (mean 22.2) years old, were recruited for the study. Participants were asked to perform the craniocervical flexion test (CCFT) in a seated position to measure deep neck flexor endurance. The thickness of DNF and SCM was assessed bilaterally and was measured using ultrasonography images at resting, contracted, pre-terminal and terminal phases of the neck muscle endurance test. Muscle contraction pattern was also observed along with the changes in muscle thickness from the resting phase to the terminal phase. Repeated-measure ANOVA was employed to compare muscle thickness bilaterally at each phase.

Results: The thickness of right and left muscles was found to be significantly different in DNF both at resting and contracted phases $(p=0.02, p<0.01$, respectively), whereas no significant difference was observed in SCM at resting or contracted phases $(p=0.59$, $p=0.18$, respectively). Thickness changes from resting to contracted phase were not significantly different both in DNF and SCM ( $p=0.18, p=0.22$, respectively). Muscle contraction patterns in right and left muscles were shown to be similar.

Conclusion: The current study, performed on (with) healthy subjects, significantly detected different right and left muscle thickness in DNF, but the muscle contraction patterns were similar in DNF and SCM bilaterally. Further study is required to investigate DNF and SCM muscle size and function in people with neck pain.
\end{abstract}

Keywords: Deep neck flexor, SCM, Ultrasonographic imaging

\section{INTRODUCTION}

Neck pain has been reported as one of the most common musculoskeletal conditions and there is an increasing prevalence rate among young adult groups. It was present in $38 \%$ of Australian subjects aged less than 40 years and many woke up with it at least once a week. ${ }^{1}$ Similar prevalence rates among young adults have been observed in other countries, ${ }^{2-4}$ and it has become a substantial socioeconomic problem. ${ }^{5}$

Neck muscles have an important role in maintaining the stability of cervical vertebrae, and it has been suggested that dysfunction of these muscles is closely associated with neck pain. For instance, weakness of the anterior cervical neck flexor muscles is thought to

Received Nov 25, 2016 Revised Dec 12, 2016

Accepted Dec 20, 2016

Corresponding author Ju-Min Song

E-mail jmsong@silla.ac.kr contribute to persistent neck pain, ${ }^{6,7}$ and decreased strength and endurance of these muscles have been observed in patients with neck pain. ${ }^{8}$ Neck muscle endurance and size including thickness have been investigated in clinical neck pain populations, and the results have been compared against asymptomatic subjects; unfortunately, muscle endurance and size have not been assessed in subclinical populations.

In general, long-lasting stimulation, such as that associated with physical training, changes the distribution of muscle fibers. Results of experimental studies that have investigated the types of muscle fibers found in anterior and posterior neck muscles showed that fiber transformations proceeded from slow oxidative to fast glycolytic among subjects with neck pain.,10

Copylight (C2016 The Korea Society of Physical Therapy

This is an Open Access article distribute under the terms of the Creative Commons Attribution Non-commercial License (Http:// creativecommons.org/license/by-nc/4.o.) which permits unrestricted non-commercial use, distribution, and reproduction in any medium, provided the original work is properly cited. 
Furthermore, it has been reported that subjects with neck pain have a higher sternocleidomastoid muscle (SCM) fatigue rate than that of control subjects. ${ }^{9,11}$ It has also been reported that individuals with neck pain have impairment of the deep neck flexor muscles (DNF). Compared to asymptomatic individuals, subjects with chronic neck pain seem to exhibit less activity in the DNF. The longus capitis (Lcap) and longus colli (Lcol) are the primary DNF involved in the support and control of the cervical curve. Morphological studies have demonstrated that the cross-sectional area of Lcol on both sides is smaller in patients with chronic neck pain than in healthy subjects. ${ }^{12}$

Rehabilitative ultrasonographic imaging has been recently used by physiotherapists to evaluate muscle structure and function. ${ }^{13}$ In addition, it can be used to measure muscle thickness and cross-sectional area when quantifying muscle atrophy or deficits in muscle activation. ${ }^{14,15}$ By using ultrasonography, it was observed that subjects with chronic neck pain have smaller increase in deep cervical flexor thickness during the craniocervical flexion test (CCFT) than asymptomatic subjects. In physiotherapy, the function of DNF muscles is commonly assessed by CCFT since they can influence cervical spine function. Real-time ultrasonographic imaging has been reported as a reliable and valid tool. Moreover, it is of relatively low cost and is easily accessible.

Due to recent trends in modern society, smartphones are becoming extremely common and widespread, and to some extent have reduced computer use. Individuals who are spending hours hunching over a computer's visual display terminal can introduce a constant and destructive physiological loads on their neck. As internet usage including smartphone use is becoming extremely high among university student populations, ${ }^{16}$ it is expected that smartphone users could represent a population with a relatively high incidence of neck pain problems related to sustained, long-term, and abnormal physiological loads on the neck. Therefore, the purpose of the study was to investigate the thickness of deep neck muscles during CCFT of university students with no current neck pain using ultrasonography images.

\section{METHODS}

\section{Subjects}

Twenty-two volunteers who were using a smartphone more than
2 hours daily participated in the study. They were included if they had no current neck or upper limb pain and/or discomfort and had no history of musculoskeletal or neurological conditions affecting daily activity functions of their neck and/or upper limbs. Prior to the study, all subjects gave their consent, and all were right handed.

\section{Measurements and Procedures}

\section{1) Deep neck flexor endurance test}

CCFT was used for measuring endurance of DNF. Subjects were instructed to perform a nodding movement generating craniocervical flexion to maximally activate the Lcol and Lcap DNF. ${ }^{17,18}$ DNF endurance time was collected using a stopwatch (in seconds). The pressure sensor of a stabilizer pressure biofeedback unit was used for screening the amount of nodding movement in five incremental levels between 22 and $30 \mathrm{mmHg}$ with $2 \mathrm{mmHg}$ progressive pressure increases. Subject should maintain an isometric contraction at the progressive pressures as an endurance task. Prior to testing, subjects were in an upright sitting posture on an examination chair with their arms resting on their thighs. After which, they were asked to assume a neutral head and neck position and were asked to maintain this position for as long as they can. The testing protocol used in this study followed the same protocols of previous studies. The CCFT has been shown to have good reliability. ${ }^{19}$

\section{2) Measurement of muscle thickness}

During the endurance test, the size of DNF and SCM was assessed using a Mylab 25GOLD ultrasound system (Esaote, Florence, Italy) with a $12 \mathrm{MHz}$ linear probe. The probe was placed longitudinally on the anterior neck, parallel to the trachea. The thickness of the SCM and DNF was measured bilaterally at the fourth cervical spine. This segmental level was chosen because it clearly exposed the target muscles under investigation, and this location is assumed to reflect the coordination between the three muscles being assessed.

Meanwhile, thickness of each muscle was measured (Figure 1) using sonography imaging which was taken from real-time measurements of the muscle endurance test at resting, contracted, preterminal, and terminal phases. The resting phase was defined as the relaxed state just before the endurance test started, the contracted phase was the starting point of the endurance test where maximum muscle contraction was observed, the pre-terminal phase was the 


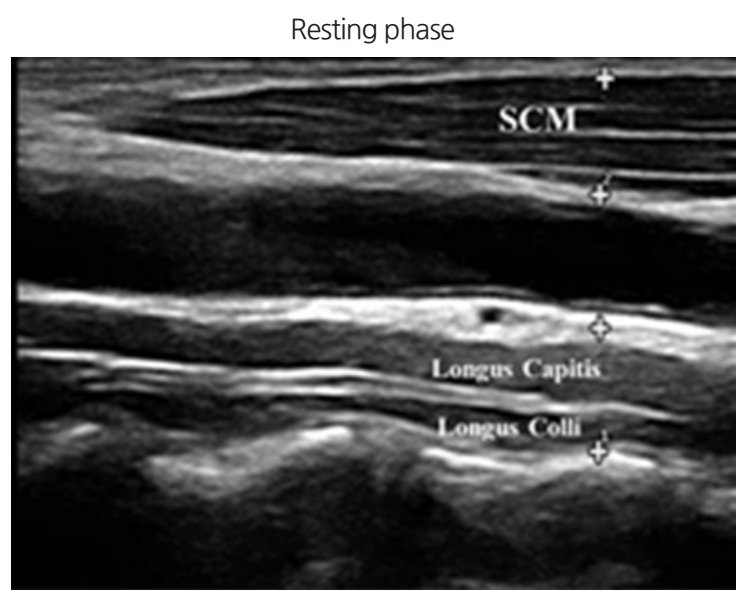

Pre-terminal phase

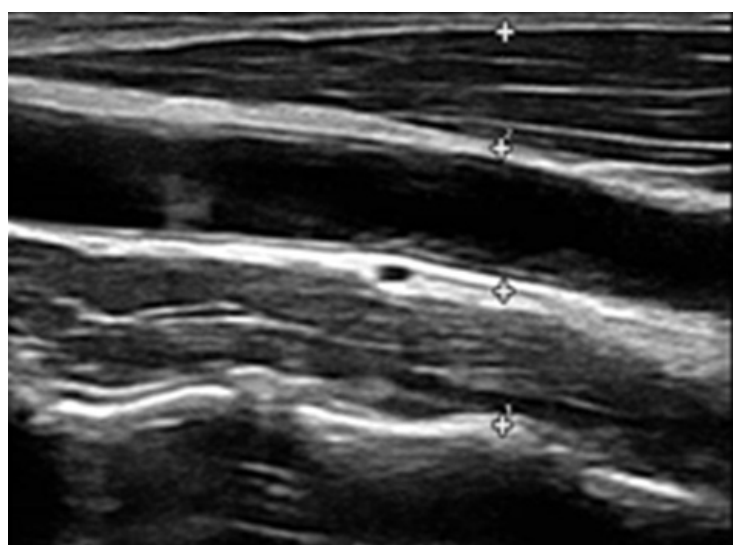

Contracted phase

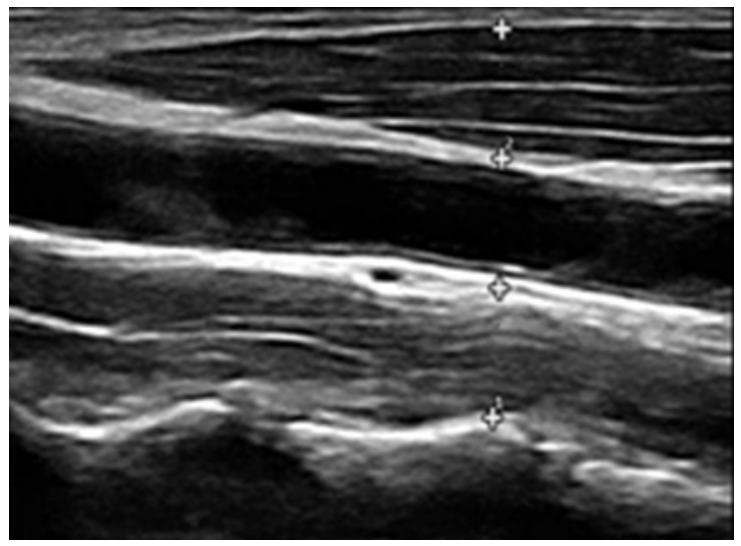

Terminal phase

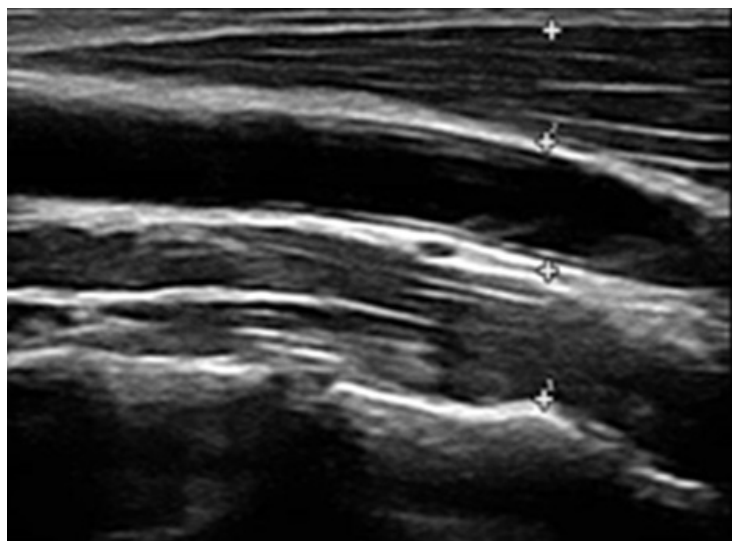

Figure 1. Representative ultrasonographic images of DNF and SCM in resting, contracted, pre-terminal, and terminal phases of CCFT. CCFT: craniocervical flexion test.

point where a subject could not hold their testing position any longer, and the terminal phase was the termination point of the endurance test. Muscle contraction pattern could be also observed along with the changes of muscle thickness from the resting phase to the terminal phase.

Reliability of neck muscle size measurements obtained by ultrasonography has been reported to be high and it has been shown, via comparison with magnetic resonance imaging results, to provide valid measurements of muscle thickness in both resting and contracted conditions. ${ }^{20}$ Measurements were taken by one examiner in order to minimize measurement bias.

\section{Analysis}

Repeated-measures ANOVA was employed to analyze the muscle function data. Mean and standard deviation of muscle thickness, endurance time, and demographic data were determined and compared.

\section{RESULTS}

Twenty-two (11 female, 11 male) healthy volunteers, aged between 20 and 25 (mean 21) years, participated in the study. Mean (standard deviation) of height and weight were $171.90(6.24) \mathrm{cm}, 62.20$ (9.93) $\mathrm{kg}$ respectively for male and $161.70(4.68) \mathrm{cm}, 52.90(5.76) \mathrm{kg}$ respectively for female. All participants were right handed. Mean (standard deviation) value of neck muscle endurance test was 83.05 (23.76) seconds. Mean and standard deviation of DNF and SCM thickness at each phase were reported in Table 1.

Lcap and Lcol sizes were measured together due to ambiguity in identifying the individual muscle boundaries, and the combined Lcap and Lcol results were grouped to form the DNF result. As for the results of the repeated-measures ANOVA for muscle thickness, DNF thickness was observed to be significantly different on two occasions when right and left DNF were compared. The right DNF was significantly thicker than the left in the resting and contracted 
Table 1. The mean (standard deviation) and p-values of deep neck flexor (DNF) and sternocleidomastoid muscle (SCM) thickness at each phase

\begin{tabular}{|c|c|c|c|c|c|}
\hline & Resting phase & Contracted phase & Pre-terminal phase & Terminal phase & $\mathrm{p}$-value \\
\hline Right DNF & $6.72(1.70)$ & $8.58(2.23)$ & $7.40(2.19)$ & $7.13(2.36)$ & $<0.01$ \\
\hline Left DNF & $5.86(1.66)$ & $7.22(2.12)$ & $6.80(1.96)$ & $6.20(2.16)$ & $<0.01$ \\
\hline Right SCM & $5.34(1.17)$ & $6.85(1.78)$ & $6.05(1.37)$ & $5.48(1.25)$ & $<0.01$ \\
\hline Left SCM & $5.10(1.40)$ & $6.19(1.50)$ & $5.47(1.16)$ & $5.13(1.09)$ & $<0.01$ \\
\hline
\end{tabular}
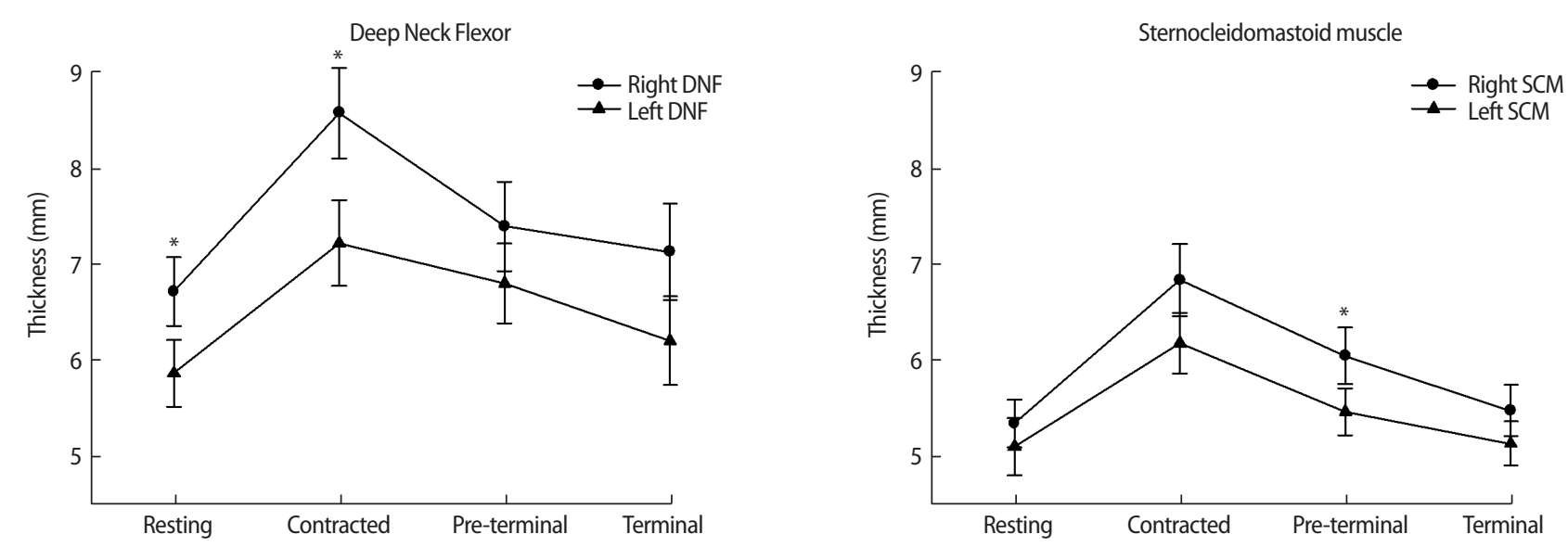

Figure 2. Muscle contraction pattern of DNF and SCM muscles during CCFT showing the changes in thickness as measured bilaterally in the resting, contracted, pre-terminal, and terminal phases. DNF: deep neck flexor, SCM: sternocleidomastoid muscle, CCFT: craniocervical flexion test. ${ }^{*} p<0.05$.

phases $(\mathrm{p}=0.02, \mathrm{p}<0.01$, respectively), but there was no significant difference in the pre-terminal and terminal phases $(p>0.05)$. The left and right SCM only exhibited a difference in thickness in the pre-terminal phase $(\mathrm{p}=0.05)$. Occasional difference was observed in both DNF and SCM. Muscle thickness in the contracted phase was significantly thicker than the resting and the terminal phases $(\mathrm{p}<0.01)$. No occasional interaction on each side was found. Changes in thickness on both sides from resting to contracted were not significantly different both in DNF and SCM ( $p=0.18, p=0.22$, respectively).

Muscle contraction pattern was observed along with the changes in muscle thickness from resting to contracted, pre-terminal and terminal phases. Muscle thickness was increased from the resting to the contracted, and then decreased gradually to the pre-terminal and the terminal phases. Changes in thickness of each muscle had similar pattern between left and right sides, as well as between DNF and SCM (Figure 2).

\section{DISCUSSION}

Ultrasonography was used to investigate changes in DNF and
SCM thickness during DNF endurance testing in resting, contracted, pre-terminal, and terminal phases in individuals with no current neck pain. Muscle thickness was observed bilaterally and it was found to be different between right and left sides at the resting and contracted phases in DNF and in the pre-terminal phase in SCM. The contracting patterns were similar in DNF and SCM.

Although no previously reported data can be directly compared to this study, the current results showed evidence of deep and superficial neck flexor activity during CCFT. In the present study, there was a tendency toward a more marked increase in thickness between the resting and contracted phases in the DNF than in the SCM. This result is similar to a previous study. ${ }^{21}$ The difference may be because DNF muscles are the main controller of neck movement during the test. Meanwhile, the contraction patterns of DNF and SCM were similar in this study, which is consistent with previous studies, where superficial muscle activity (including SCM) was observed to increase to a lesser degree in individuals with no symptoms compared to a greater degree in individuals with neck pain and/or headache. ${ }^{22,23}$ Many studies have used CCFT to assess neck muscle functions in people with neck pain and these studies reported similar findings of increased activation of superficial muscles in 
a chronic neck pain population. The observed increase in superficial muscle activity may be interpreted as a form of compensation for reduced activation of DNF. ${ }^{6}$

An interesting observation in this study was the bilateral asymmetry of neck muscle thickness even though the participants in the study reported no current neck pain and/or discomfort. The right DNF was thicker than the left when the neck was relaxed; however, SCM sizes were not bilaterally different. There were no previous data available on DNF thickness asymmetry to which the current results could be compared. It is probable that the observed asymmetry was due to habitual head movements associated with handedness. The current study participants were smartphone users and all were right handed. The small visual display terminal of smartphones may have affected subject head movement, and such movement might be related to subject handedness. For example, righthanded subjects when using a smartphone may be flexing and/or rotating their neck to the right more often than to the left side or the middle. Therefore, neck flexor muscles on either side may work differently during the conduct of daily activities including smartphone usage. The right SCM was only observed to be thicker than the left at the pre-terminal phase, which may reflect activation compensation for right DNF fatigue.

The current study participants were regular smartphone users who may spend hours in habitual postures such as a head forward posture. Such postures may contribute to DNF weakness. The study on participants' neck muscle contraction pattern was not similar to that of the clinical neck pain population. No pain data was reported in the current study, however, the group may have had neck pain/ discomfort.16 No data have been previously reported on the activation of deep and superficial neck muscles during CCFT in subjects who have more likely been experiencing neck pain/discomfort but not necessarily having current neck pain, which might be indicating a sub-clinical neck pain population. Subjects in this group may change their "pain- or discomfort-generating" body postures or activities to a more comfortable ones as their self-management strategy and they are less likely looking for professional treatments. Since neck structures including DNF are vulnerable from a constant physiological load during habitual activities like dominant hand and smartphone usage, it is suggested that they should be strengthened and an anatomically recommended head posture should be maintained in an upright position as a form of self-management.
This self-management approach, when done properly and consistently, may be sufficient to prevent the development of severe neck pain.

The results of this study provided indications of possible research topics that may assist in the development of neck muscle testing, prevention and rehabilitation, as well as muscle impairment monitoring in neck pain populations. For example, research is required to investigate the right and left neck muscle contraction patterns in people with and without neck pain. In addition, a longitudinal study into muscle asymmetry may indicate how handedness or smartphone use can affect neck pain generation and/or cervical spine dysfunction.

\section{REFERENCES}

1. Gordon SJ, Trott P, Grimmer KA. Waking cervical pain and stiffness, headache, scapular or arm pain: gender and age effects. J Physiother. 2002;48(1):9-15.

2. Guez M, Hildingsson C, Nilsson M et al. The prevalence of neck pain: a population-based study from northern sweden. Acta Orthop Scand. 2002;73(4):455-9.

3. Strine TW, Hootman JM. Us national prevalnce and correlates of low back and neck pain among adults. Arthritis Rheum. 2007;57(4):656-65.

4. Chiu TTW, Lam PKW. The prevalence of and risk factors for neck pain and upper limb pain among secondary school teachers in Hong Kong. J Occup Rehabil. 2007;17(1):19-32.

5. Cote P, Kristman V, Vidmar M et al. The prevalence and incidence of work absenteeism involving neck pain: a cohort of ontario lost-time claimants. J Manipulative Physiol Ther. 2009;32(2 Suppl):S219-26.

6. Jull G, Falla D. Does increased superficial neck flexor activity in the craniocervical flexion test reflect reduced deep flexor activity in people with neck pain? Man Ther. 2016;25:43-7.

7. Falla D, O'Leary S, Farina D et al. The change in deep cervical flexor activity after training is associated with the degree of pain reduction in $\mathrm{pa}$ tients with chronic neck pain. Clin J Pain. 2012;28(7):628-34.

8. Edmondston S, Bjornsdottir G, Palsson T et al. Endurance and fatigue characteristics of the neck flexor and extensor muscles during isometric tests in patients with postural neck pain. Man Ther. 2011;16(4):332-8.

9. Falla D, Rainoldi A, Merletti R et al. Myoelectric manifestations of sternocleidomastoid and anterior scalene muscle fatigue in chronic neck pain patients. Clin Neurophysiol. 2003;114(3):488-95.

10. Uhlig Y, Weber BR, Grob D et al. Fiber composition and fiber transformations in neck muscles of patients with dysfunction of the cervical spine. J Orthop Res. 1995;13(2):240-9.

11. Gogia PP, Sabbahi MA. Electromyographic analysis of neck muscle fatigue in patients with osteoarthritis of the cervical spine. Spine. 1994; 19(5):502-6.

12. Javanshir K, Rezasoltani A, Mohseni-Bandpei MA et al. Ultrasound assessment of bilateral longus colli muscles in subjects with chronic bilateral neck pain. Am J Phys Med Rehabil. 2011;90(4):293-301. 
13. Whittaker JL, Stokes M. Ultrasound imaging and muscle function. J Orthop Sports Phys Ther. 2011;41(8):572-80.

14. Jesus FMR, Ferreira PH, Ferreira ML. Ultrasonographic measurement of neck muscle recruitment: a preliminary investigation. J Manipulative Physiol Ther. 2008;16(2):89-92.

15. Ishida H, Suehiro T, Kurozumi C et al. Ultrasound imaging of the diagonal dimension of the deep cervical flexor muscles: a reliability study on healthy subjects. J Bodyw Mov Ther. 2015;19(3):417-20.

16. Lee H. Neck pain and functioning in daily activities associated with smartphone usage. J Kor Phys Ther. 2016;28(3):183-8.

17. Falla D, O'Leary S, Fagan A et al. Recruitment of the deep cervical flexor muscles during a postural-correction exercise performed in sitting. Man Ther. 2007;12(2):139-43.

18. Cagnie B, D'Hooge R, Achten E et al. A magnetic resonance imaging investigation into the function of the deep cervical flexors during the performance of craniocervical flexion. J Manipulative Physiol Ther. 2010;
33(4):286-91.

19. Olson LE, Millar AL, Dunker J et al. Reliability of a clinical test for deep cervical flexor endurance. J Manipulative Physiol Ther. 2006;29(2):1348 .

20. Cagnie B, Derese E, Vandamme L et al. Validity and reliability of ultrasonography for the longus colli in asymptomatic subjects. Man Ther. 2009;14(4):421-6.

21. Falla D, Jull G, Dall'Alba P et al. An electromyographic analysis of the deep cervical flexor muscles in performance of craniocervical flexion. Phys Ther. 2003;83(10):899-906.

22. Javanshir K, Amiri M, Mohseni-Bandpei MA et al. Ultrasonography of the cervical muscles: a critical review of the literature. J Manipulative Physiol Ther. 2010;33(8):630-7.

23. Zito G, Jull G, Story I. Clinical tests of musculoskeletal dysfunction in the diagnosis of cervicogenic headache. Man Ther. 2006;11(2):118-29. 